General Mathematics Vol. 28, No. 2 (2020), 81-85

DOI: $10.2478 / \mathrm{gm}-2020-0017$

sciendo

\title{
Differences and quotients of positive linear operators ${ }^{1}$
}

\author{
Sever Hodiş
}

\begin{abstract}
We consider the classical Szász-Mirakyan and Szász-Mirakyan-Durrmeyer operators, as well as a Kantorovich modification and a discrete version of it. The images of exponential functions under these operators are determined. We establish estimates involving differences and quotients of these images.
\end{abstract}

2010 Mathematics Subject Classification: 41A36.

Key words and phrases: differences of positive linear operators, quotients of positive linear operators, images of exponential functions.

\section{Introduction}

For $n \geq 1$ and $k=0,1, \ldots$, let $s_{n, k}(x):=e^{-n x} \frac{(n x)^{k}}{k !}, x \in[0, \infty)$.

Let

$$
\mathbb{S}_{n}(f ; x):=\sum_{k=0}^{\infty} f\left(\frac{k}{n}\right) s_{n, k}(x),
$$

be the Szász-Mirakyan operators, and

$$
\mathbb{D}(f ; x):=n \sum_{k=0}^{\infty} s_{n, k}(x) \int_{0}^{\infty} f(t) s_{n, k}(t) d t, x \in[0, \infty),
$$

the Szász-Mirakyan-Durrmeyer operators. We consider those functions $f \in C[0, \infty)$ for which the integrals and the series are convergent.

The operators

$$
\mathbb{K}_{n}(f ; x):=\sum_{j=0}^{\infty}\left(n \int_{\frac{j}{n}}^{\frac{j+1}{n}} f(t) d t\right) e^{-n x} \frac{(n x)^{j}}{j !}, x \geq 0,
$$

\footnotetext{
${ }^{1}$ Received 26 July, 2020

Accepted for publication (in revised form) 20 September, 2020
} 
are Kantorovich-type modifications of $\mathbb{K}_{n}$.

Moreover,

$$
D_{n}(f ; x):=\sum_{j=0}^{n} f\left(\frac{2 j+1}{2 n}\right) e^{-n x} \frac{(n x)^{j}}{j !}, x \geq 0,
$$

is the sequence of discrete operators associated with $\mathbb{K}_{n}$.

Details concerning these operators can be found in [1] and [2].

Let $n \geq 1$ be given. In Section 2 we shall determine the images of the functions $f_{\lambda}(t):=e^{\overline{\lambda t}}, t \geq 0,0<\lambda<n$, under the operators $\mathbb{S}_{n}, \mathbb{D}_{n}, \mathbb{K}_{n}$ and $D_{n}$.

Estimates involving differences and quotients of these images will be presented in Section 3.

\section{Images of the function $f_{\lambda}$}

Let $\theta:=\frac{\lambda}{n} \in(0,1)$.

Theorem 1 With the above notation we have for $x \geq 0$,

$$
\text { i) } \mathbb{S}_{n}\left(f_{\lambda} ; x\right)=\exp \left(\frac{e^{\theta}-1}{\theta} \lambda x\right)
$$

$$
\text { ii) } \mathbb{D}_{n}\left(f_{\lambda} ; x\right)=\frac{1}{1-\theta} \exp \left(\frac{1}{1-\theta} \lambda x\right)
$$

$$
\text { iii) } \mathbb{K}_{n}\left(f_{\lambda} ; x\right)=\frac{e^{\theta}-1}{\theta} \exp \left(\frac{e^{\theta}-1}{\theta} \lambda x\right) \text {, }
$$

$$
\text { iv) } D_{n}\left(f_{\lambda} ; x\right)=\left(\exp \frac{\theta}{2}\right) \exp \left(\frac{e^{\theta}-1}{\theta} \lambda x\right) \text {. }
$$

Proof. i) According to (1) we have

$$
\begin{aligned}
\mathbb{S}_{n}\left(f_{\lambda} ; x\right) & =\sum_{k=0}^{\infty} e^{\frac{\lambda k}{n}} e^{-n x} \frac{(n x)^{k}}{k !} \\
& =e^{-n x} \sum_{k=0}^{\infty} \frac{\left(n x e^{\theta}\right)^{k}}{k !} \\
& =e^{-n x} e^{n x e^{\theta}} \\
& =\exp \left(n x\left(e^{\theta}-1\right)\right) \\
& =\exp \left(\frac{e^{\theta}-1}{\theta} \lambda x\right) .
\end{aligned}
$$


ii) Now we use (1) to get

$$
\mathbb{D}\left(f_{\lambda} ; x\right)=n \sum_{k=0}^{\infty} e^{-n x} \frac{(n x)^{k}}{k !} \int_{0}^{\infty} e^{\lambda t} e^{-n t} \frac{(n t)^{k}}{k !} d t .
$$

The last integral is in fact

$$
\begin{aligned}
\frac{n^{k}}{k !} \int_{0}^{\infty} e^{-(n-\lambda) t} t^{k} d t & =\frac{n^{k}}{k !} \int_{0}^{\infty} e^{-s} \frac{s^{k}}{(n-\lambda)^{k+1}} d s \\
& =\frac{n^{k}}{(n-\lambda)^{k+1}} \frac{1}{k !} \Gamma(k+1) \\
& =\frac{n^{k}}{(n-\lambda)^{k+1}} .
\end{aligned}
$$

Consequently,

$$
\begin{aligned}
\mathbb{D}_{n}\left(f_{\lambda} ; x\right) & =n e^{-n x} \sum_{k=0}^{\infty} \frac{(n x)^{k}}{k !} \frac{n^{k}}{(n-\lambda)^{k+1}} \\
& =\frac{n}{n-\lambda} e^{-n x} \exp \left(\frac{n^{2} x}{n-\lambda}\right) \\
& =\frac{1}{1-\theta} \exp \left(\frac{n^{2} x}{n-\lambda}-n x\right) \\
& =\frac{1}{1-\theta} \exp \left(\frac{1}{1-\theta} \lambda x\right) .
\end{aligned}
$$

iii) Using (3) we obtain

$$
\begin{aligned}
\mathbb{K}_{n}\left(f_{\lambda} ; x\right) & =\frac{n e^{-n x}}{\lambda} \sum_{j=0}^{\infty}\left(e^{\frac{j+1}{n} \lambda}-e^{\frac{j}{n} \lambda}\right) \frac{(n x)^{j}}{j !} \\
& =\frac{e^{-n x}}{\theta} \sum_{j=0}^{\infty}\left(e^{(j+1) \theta}-e^{j \theta}\right) \frac{(n x)^{j}}{j !} \\
& =\frac{e^{-n x}}{\theta}\left(e^{\theta}-1\right) \sum_{j=0}^{\infty} \frac{\left(n x e^{\theta}\right)^{j}}{j !} \\
& =\frac{e^{\theta}-1}{\theta} e^{-n x} e^{n x e^{\theta}} \\
& =\frac{e^{\theta}-1}{\theta} \exp \left(\frac{e^{\theta}-1}{\theta} \lambda x\right) .
\end{aligned}
$$


iv) According to (4),

$$
\begin{aligned}
D_{n}\left(f_{\lambda} ; x\right) & =\sum_{j=0}^{\infty}\left(\exp \left(\frac{2 j+1}{2 n} \lambda-n x\right)\right) \frac{(n x)^{j}}{j !} \\
& =\sum_{j=0}^{\infty}\left(\exp \frac{j \lambda}{n} \exp \left(\frac{\lambda}{2 n}-n x\right)\right) \frac{(n x)^{j}}{j !} \\
& =\left(\exp \left(\frac{\theta}{2}-n x\right)\right) \exp \left(n x e^{\theta}\right) \\
& =e^{\frac{\theta}{2}} \exp \left(n x\left(e^{\theta}-1\right)\right) \\
& =e^{\frac{\theta}{2}} \exp \left(\frac{e^{\theta}-1}{\theta} \lambda x\right) .
\end{aligned}
$$

\section{Differences and quotients of images}

Due to the structure of equations (5)-(8), it is more convenient to study quotients of operators and differences of operators acting on exponential functions. For example,

$$
\frac{\mathbb{K}_{n}\left(f_{\lambda} ; x\right)}{\mathbb{S}_{n}\left(f_{\lambda} ; x\right)}=\frac{n}{\lambda}\left(e^{\frac{\lambda}{n}}-1\right), x \geq 0
$$

and

$$
\frac{D_{n}\left(f_{\lambda} ; x\right)}{\mathbb{S}_{n}\left(f_{\lambda} ; x\right)}=e^{\frac{\lambda}{2 n}}, x \geq 0 .
$$

For a fixed $\lambda>0$, both quotients tend to 1 when $n \rightarrow \infty$.

Let us mention also the following result.

Proposition 1 For $x \geq 0$ and $0<\lambda<n$,

$$
0 \leq \frac{\mathbb{K}_{n}\left(f_{\lambda} ; x\right)-D_{n}\left(f_{\lambda} ; x\right)}{\mathbb{S}_{n}\left(f_{\lambda} ; x\right)} \leq(e-\sqrt{e}-1)\left(\frac{\lambda}{n}\right)^{2},
$$

and

$$
1 \leq \frac{\mathbb{D}_{n}\left(f_{\lambda} ; x\right)}{\mathbb{K}_{n}\left(f_{\lambda} ; x\right)} \leq\left(\frac{n}{n-\lambda}\right)^{\frac{n \lambda x}{n-\lambda}+1} .
$$

Proof. Using (9) and (10) we have

$$
\begin{aligned}
\frac{\mathbb{K}_{n}\left(f_{\lambda} ; x\right)-D_{n}\left(f_{\lambda} ; x\right)}{\mathbb{S}_{n}\left(f_{\lambda} ; x\right)} & =\frac{e^{\theta}-1}{\theta}-e^{\frac{\theta}{2}} \\
& =\sum_{j=2}^{\infty}\left(\frac{1}{(j+1) !}-\frac{1}{j ! 2^{j}}\right) \theta^{j} \\
& \leq \theta^{2} \sum_{j=2}^{\infty}\left(\frac{1}{(j+1) !}-\frac{1}{j ! 2^{j}}\right) \\
& =\theta^{2}(e-\sqrt{e}-1),
\end{aligned}
$$


and so (11) is proved.

To prove (12), let us use the notation

$$
\begin{gathered}
a:=\frac{e^{\theta}-1}{\theta}=1+\frac{\theta}{2 !}+\frac{\theta^{2}}{3 !}+\ldots, \\
b:=\frac{1}{1-\theta}=1+\theta+\theta^{2}+\ldots .
\end{gathered}
$$

Then $1<a<b$ and

$$
\begin{aligned}
\frac{\mathbb{D}_{n}\left(f_{\lambda} ; x\right)}{\mathbb{K}_{n}\left(f_{\lambda} ; x\right)} & =\frac{a}{b} \exp ((b-a) \lambda x) \\
& =\frac{b}{a} \exp \left(\frac{b-a}{\log \frac{a}{b}} \lambda x \log \frac{b}{a}\right) \\
& \leq \frac{b}{a} \exp \left(b \lambda x \log \frac{b}{a}\right) \\
& =\left(\frac{b}{a}\right)^{b \lambda x+1} \leq b^{b \lambda x+1} \\
& =\left(\frac{1}{1-\theta}\right)^{\frac{\lambda x}{1-\theta}+1} \\
& =\left(\frac{n}{n-\lambda}\right)^{\frac{n \lambda x}{n-\lambda}+1} .
\end{aligned}
$$

Remark 1 According to (11) and (12), the corresponding quotients tend to 0, respectively to 1 , when $n$ goes to infinity.

\section{References}

[1] M. Heilmann, F. Nasaireh, I. Raşa, Discrete Operators associated with Linking Operators, arXiv:1808.07239v1.

[2] I. Raşa, Discrete operators associated with certain integral operators, Stud. Univ. Babeş-Bolyai Math., vol. 56, no. 2, 2011, 537-544.

\section{Sever Hodiş}

Technical University of Cluj-Napoca

Department of Mathematics

Str. Memorandumului, nr. 28, Cluj-Napoca, Romania e-mail: hodissever@gmail.com 